\title{
Sobre la constitución química del agua de amasado y las teorías de la hidratación del cemento
}

P. KITTL* y A. GOLDSCHMIDT**

\author{
R E S U M E N
}

Se estudia la composición del agua de amasado $(a / c=0,5)$ de un cemento comercial, obteniendo que a los 10 minutos del comienzo se trata de una solución muy concentrada de $\mathrm{KOH}$ y $\mathrm{NaOH}$ con algo de $\mathrm{CaOH}, \mathrm{SO}_{3} \mathrm{Ca}, \mathrm{Al}, \mathrm{Fe}$ y vestigios de Si. La composición del agua de amasado a las 24 horas es similar. Evaporando estas soluciones no se encontró ninguna zona de composición del tipo $\mathrm{CaO} . \mathrm{H}_{2} \mathrm{O}_{\text {S }} \mathrm{SiO}_{2}$ correspondiente a la familia de las tobermoritas $y$, por lo tanto, esto se inclina fuertemente a favor de la teoría topoquímica de la hidratación.

\section{N T R O D U C C I O N}

La hidratación del cemento puede describirse a través de dos mecanismos, que corresponden: a la escuela de Le Chatelier (1), sostenida principalmente por Williamson (2) y Dron en la actualidad (3); y la topoquímica, sostenida principalmente por Kapranov (4), Kittl, Castro (5) y otros.

El mecanismo propuesto por Le Chatelier y Williamson implica una constante disolución y precipitación de los productos hidratados a partir de una solución sobresaturada por un aumento de temperatura producido porque la hidratación es una reacción exotérmica. Este mecanismo implica que toda la masa a hidratase se debe disolver y luego precipitar. Pero por observaciones experimentales (2), los productos de hidratación se forman alrededor de los granos de clínker y esta capa bloquearía inmediatamente el mecanismo de hidratación. Entonces necesariamente hay que aceptar, incluso dentro de la idea de Le Chatelier, que debe haber un flujo de agua hacia la interfase clínker/material hidratado y un flujo de clínker disuelto hacia el exterior. El crecimiento se realizaría luego a partir de la interfase agua/material hidratado.

\footnotetext{
* Laboratorio de Microscopía Elctrónica.

Instituto de Investigaciones y Ensayos de Materiales (IDIEM) (Departamento Ciencias de los Materiales), Facultad de Ciencias Fisicas y Matemáticas, Universidad de Chile, Casilla N.0 1.420, Santiago, Chile.

** Laboratorio de Microanálisis.

Departamento de Geologia, Facultad de Ciencias Físicas y Matemáticas, Universidad de Chile, Santiago, Chile.
} 
El mecanismo topoquímico consiste en un flujo de agua a través de la capa hidratada y un crecimiento del material hidratado, a partir de la interfase clínker/material hidratado. Por supuesto que los materiales solubles que se producen, como el $\mathrm{CaOH}$, pueden precipitar a partir de una solución saturada.

El objeto del presente trabajo es buscar en el agua de amasado los compuestos que pueden precipitar en un material hidratado, como el $\mathrm{CaO}_{2} \mathrm{H}_{2} \mathrm{O} \cdot \mathrm{SiO}_{2}$, de la familia de las tobermoritas o de los silicatos de calcio hidratados.

\section{PREPARACION DE LAS MUESTRAS}

Se utilizó un cemento comercial (supercemento Melón) con un análisis químico aproximado de:

$$
\begin{array}{lll}
19,26 \% \text { de } \mathrm{SiO}_{2}, & 63,27 \% \text { de } \mathrm{CaO}, & 5,55 \% \text { de } \mathrm{Al}_{2} \mathrm{O}_{3}, \\
2,88 \% \text { de } \mathrm{Fe}_{2} \mathrm{O}_{3}, & 2,86 \% \text { de } \mathrm{SO}_{3}, & 1,97 \% \text { de } \mathrm{MgO}, \\
1,01 \% \text { de } \mathrm{K}_{2} \mathrm{O}, & 0,61 \% \text { de } \mathrm{Na}_{2} \mathrm{O}, & 0,65 \% \text { de } \mathrm{TiO}_{2}, \\
0,17 \% \text { de } \mathrm{MnO}_{2} \text { y } & 0,10 \% \text { de } \mathrm{P}_{2} \mathrm{O}_{5} * &
\end{array}
$$

Este cemento se mezcló con agua $(a / c=0,5)$ durante 3 minutos y se le extrajo el agua de amasado por prensado en molde de hierro, recogiéndose la solución en una pipeta de plástico que se guardó en un frasco del mismo material. Esta solución se filtró para eliminar partículas solubles. La solución se depositó sobre una base de Pt para evaporar el $\mathrm{H}_{2} \mathrm{O}$ y observarla en la microsonda.

Otra solución se obtuvo a partir de una muestra del mismo cemento fraguada 24 horas, en una bolsa cerrada de plástico, pulverizándola y prensándola a $1.000 \mathrm{~kg} / \mathrm{cm}^{2}$ en un molde de hierro, con lo cual se pudo extraer $1 \mathrm{~cm}^{3} / 100 \mathrm{~g}$ de cemento. La solución se recogió y preparó de la misma manera que la solución anterior.

Se analizaron 20 muestras de cada solución en la microsonda y cada una se verificó en el equipo de absorción atómica**.

\section{RESULTADOS EXPERIMENTALES}

Se examinaron las muestras con una microsonda CAMECA MS 46, usando un haz puntual de $2 \mu \mathrm{m}$ de diámetro. En general la muestra (fig. 1) tenía una distribución uniforme y abundante de $\mathrm{K}, \mathrm{Na}$, Ca y $\mathrm{S}$. Esto corresponde a $\mathrm{KOH}, \mathrm{NaOH}$ y $\mathrm{CaSO}_{3}$ que son solubles, siendo el $\mathrm{K}$ lo más abundante. En esa masa se detectó la presencia de puntos donde además existían $\mathrm{Al}$, Si y $\mathrm{Fe}$ en diferentes proporciones; estos puntos se destacaban en el microscopio óptico como de diferente coloración a la masa general, pero eran sumamente escasos, un $2 \%$ de la masa total, y con una dimensión aproximada media de $20 \mu \mathrm{m} \times$ $\times 20 \mu \mathrm{m}$. Los resultados son válidos para las dos soluciones.

\footnotetext{
* Análisis facilitado por el Sr. J. Arribada y la Sra. J. Vilaró de la Sección Investigaciones Químicas del IDIEM.

** Análisis hecho por el Laboratorio de Absorción Atómica del Departamento de Minas de la Facultad de Ciencias Físicas y Matemáticas, Universidad de Chile.
} 


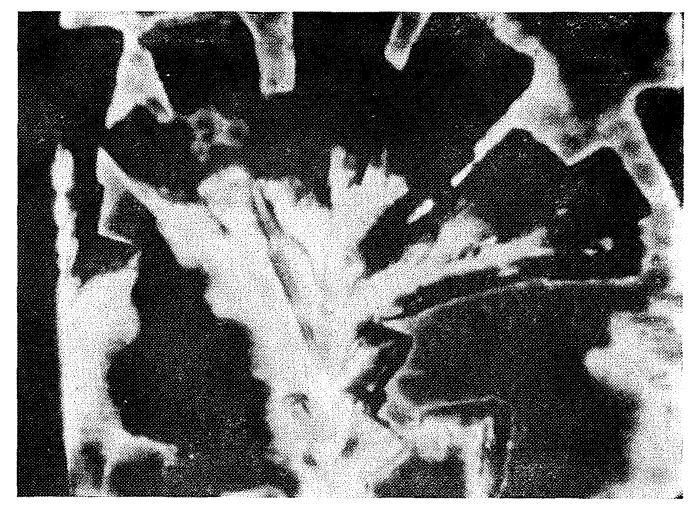

Fig. 1.-Cristales: Obtenidos por evaporación del agua de amasado de cemento, 10 minutos después de su comienzo, observados en el microscopio de absorción $(50 \mu \mathrm{m} \simeq 1 \mathrm{~cm})$.

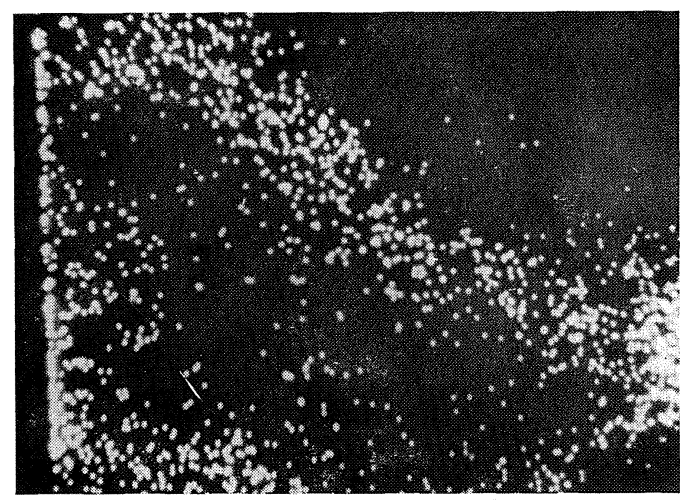

Fig. 3.-Distribución del $A l$. La misma zona que la figura anterior.

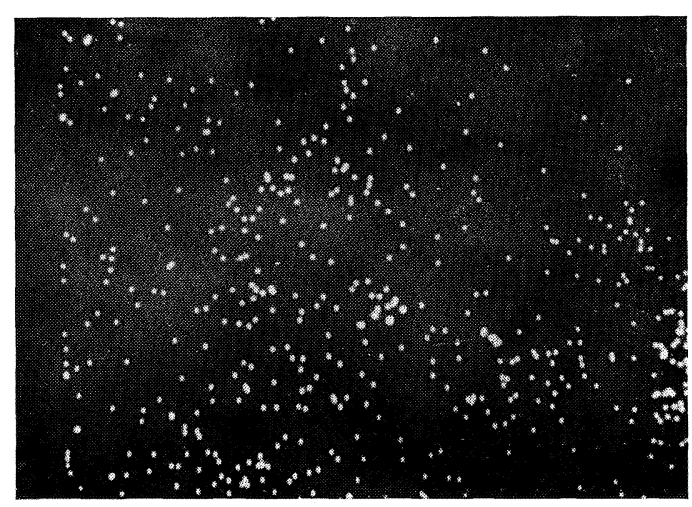

Fig. 5.-Distribución del Fe en la misma zona que las figuras $2,3 y$. Nótese la fuerte correlación sílico aluminosa, típica de los compuestos o mono y trisilico aluminatos de calcio y del mono sulfo aluminato cálcico (fígs. 2-3). Es notoria la escasez de calcio $y$ hierro (figs. 4-5).

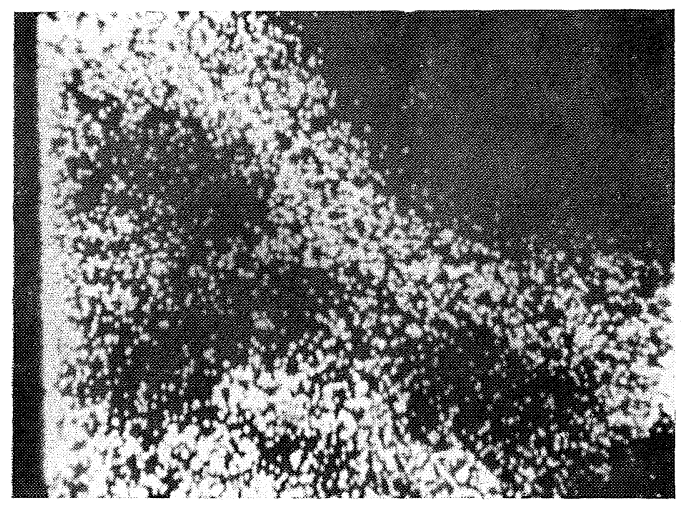

Fig. 2.-Distribución del silicio en una zona de un precipitado a partir de $\mathrm{H}_{2} \mathrm{O}$ de amasado del cemento, 10 minutos después de su comienzo (ancho $30 \mu \mathrm{m}$ ).

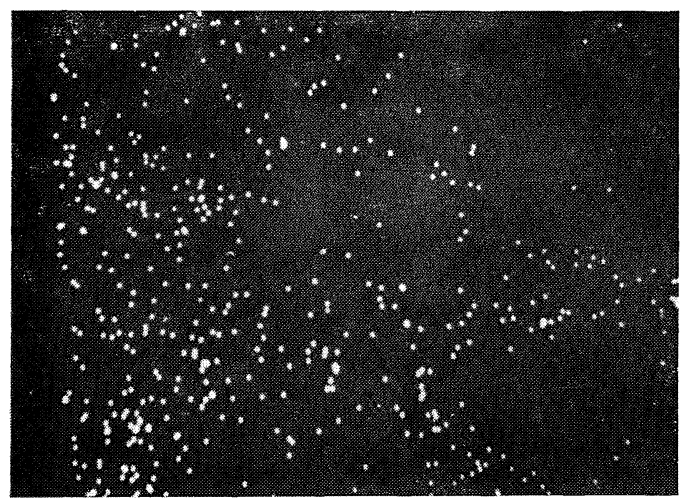

Fig. 4.-Distribución del Ca en la misma zona que las figuras 2 y 3 .

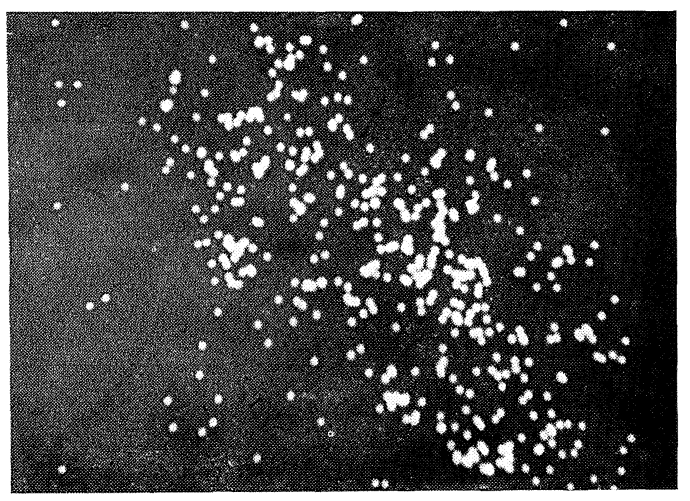

Fig. 6.-Distribución del aluminio en una zona de precipitado, a partir del agua de amasado del cemento, 10 minutos después de su comienzo (ancho $30 \mu \mathrm{m})$.

Estos puntos singulares de área media aproximada de $20 \mu \mathrm{m} \times 20 \mu \mathrm{m}$, fueron estudiados con bastante detalle con el barrido electrónico, observándose las distribuciones de $\mathrm{Ca}, \mathrm{Al}$, $\mathrm{Si}$, Fe y S (figs. 2, 3, 4, 5, 6, 7 y 8). Se pudo comprobar que entre los elementos $\mathrm{Al}$, $\mathrm{Si}$, 
$\mathrm{Ca}, \mathrm{S}$ no existe la correlación aislada Ca-Si (figs. 10-11); además siempre que hay la asociación $\mathrm{Si}-\mathrm{Ca}$ está también presente el $\mathrm{Al}$ y algunas veces el $\mathrm{S}$, siendo la correlación más fuerte la $\mathrm{Si}-\mathrm{Al}$ (figs. 2, 3, 4 y 5).

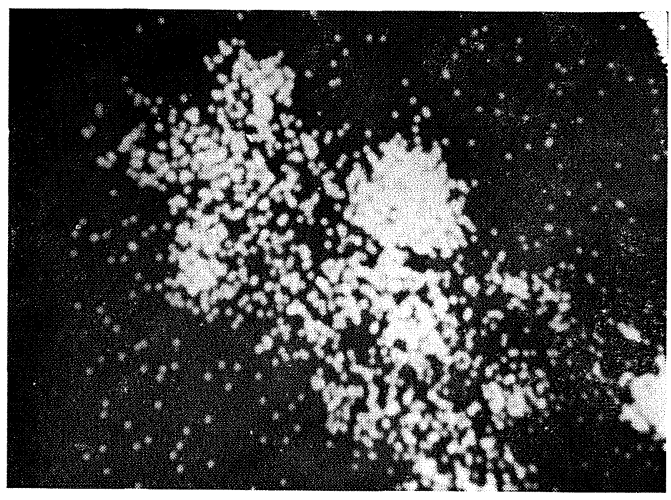

Fig. 7.-Distribución del silicio en la misma zona que la figura 6.

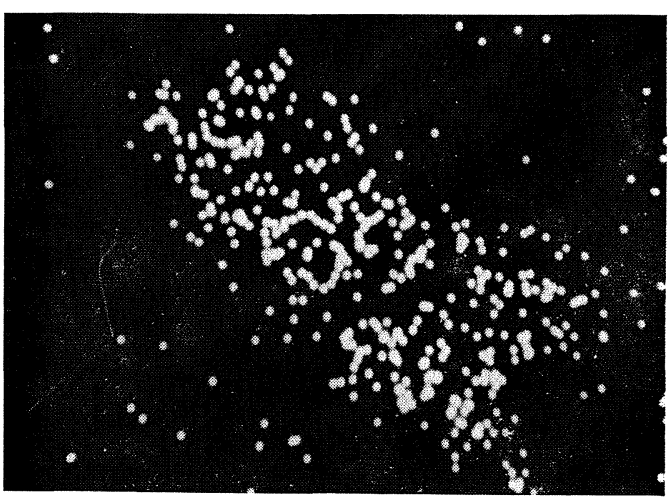

Fig. 8.-Distribución del calcio en la misma zona que las figuras 6 y $\%$. Nótese la carencia de calcio (fig. 8) en la zona de mayor intensidad de silicio (fig. 7 ).

Por absorción atómica se comprobó en el agua de amasado la presencia de $9,9 \mathrm{~g} / \mathrm{l}$ de $\mathrm{K}, 1,3 \mathrm{~g} / \mathrm{l}$ de $\mathrm{Ca}$ y $0,016 \mathrm{~g} / \mathrm{l}$ de $\mathrm{Si}$ en la muestra tomada a los 10 minutos. En la muestra de agua de amasado tomada a las 24 horas se determinó $7,2 \mathrm{~g} / l$ de $\mathrm{K}, 0,06 \mathrm{~g} / l$ de $\mathrm{Ca} \mathrm{y}$ $0,04 \mathrm{~g} / l$ de Si. Los datos correspondientes al Si dan sólo una idea del orden de magnitud, porque están afectados por errores importantes debidos a la pequeñez de la concentración.

\section{INTERPRETACION DE LOS RESULTADOS}

La presencia de un fondo común de $\mathrm{K}, \mathrm{Na}$ y $\mathrm{SO}_{3}$ corresponde al paso a solución del $\mathrm{K}_{2} \mathrm{O}$ y del $\mathrm{Na}_{2} \mathrm{O}$ presentes en el clínker y el agregado de $\mathrm{CaSO}_{3}$, que es también soluble. En presencia de $\mathrm{KOH}$ y del $\mathrm{NaOH}$ el $\mathrm{SiO}_{2}$ puede ser disuelto en una cantidad como la que se determinó por absorción atómica. Probablemente en la solución existen iones complejos, pero lo importante es que al precipitar ninguno de ellos forma la sola asociación $\mathrm{Ca}-\mathrm{Si}$. Las asociaciones existentes entre dos elementos $\mathrm{Ca}, \mathrm{Al}$, Si y $\mathrm{S}$ deben corresponder a $(\mathrm{CaO})_{3} \mathrm{Al}_{2} \mathrm{O}_{3} \cdot \mathrm{CaSiO}_{3} \cdot 12 \mathrm{H}_{2} \mathrm{O},(\mathrm{CaO})_{3} \mathrm{Al}_{2} \mathrm{O}_{3} \cdot 3 \mathrm{CaSiO}_{3} \cdot 31 \mathrm{H}_{2} \mathrm{O}$ o $3 \mathrm{CaO} \cdot \mathrm{Al}_{2} \mathrm{O}_{3} \cdot \mathrm{CaSO}_{4}$. $.12 \mathrm{H}_{2} \mathrm{O}$, que son, respectivamente, al monosilicoaluminato de calcio, al trisilicoaluminato de calcio y al monosulfoaluminato de calcio, los dos primeros encontrados por Flint y Wells (6). Se determinaron también partículas de $\mathrm{CaOH}$ precipitadas. La falta de una asoción Ca-Si aisladas (figs. 10 y 11) implica la inexistencia del silicato de calcio hidratado, del tipo $\mathrm{CaO} \cdot \mathrm{H}_{2} \mathrm{O} . \mathrm{SiO}_{2}$, de la familia de la tobermorita.

La falta de precipitados del tipo de la tobermorita, a partir del agua de amasado, es un fuerte argumento en contra de la teoría de su formación a partir de una solución sobresaturada, según Le Chatelier, y tiende a confirmar la i.dea que procede de un proceso del tipo topoquímico. Este proceso podría esquematizarse como una disolución de $\mathrm{CaO}$ del silicato di y tricálcico y un posterior ataque puzolánico, sobre el esqueleto de $\mathrm{SiO}_{2}$, para formar el silicato de calcio hidratado. 


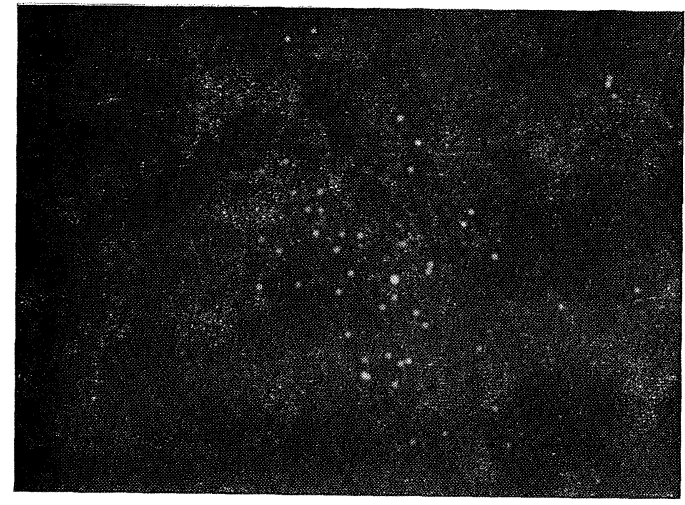

Fig. 9.-Distribución del hierro en la misma zona que las figuras $6,7,8$.

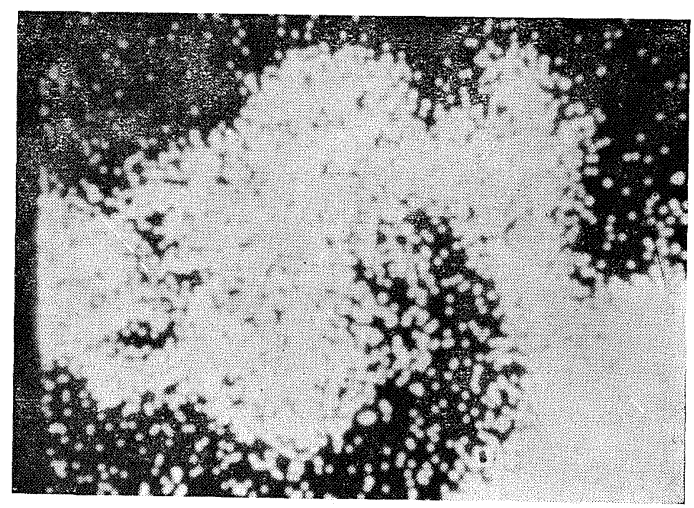

Fig. 11.-Distribución del Ca en la misma zona que la figura 10.

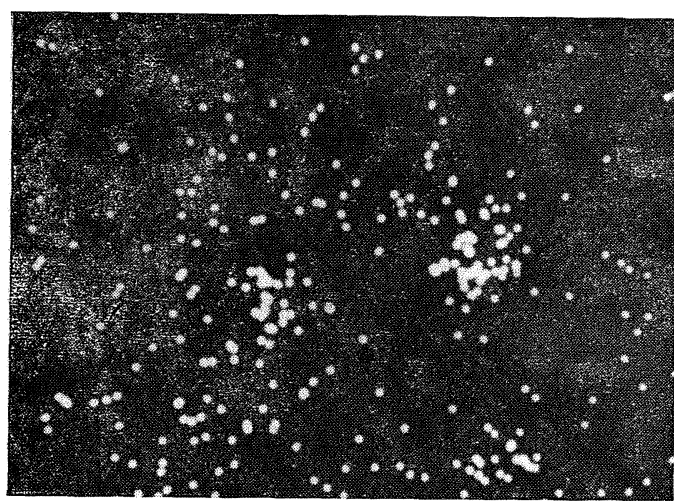

Fig. 10.-Distribución del silicio en una zona de precipitado a partir del $\mathrm{H}_{2} \mathrm{O}$ de amasado del cemento, 10 minutos después de su comienzo (ancho $30 \mu \mathrm{m})$.

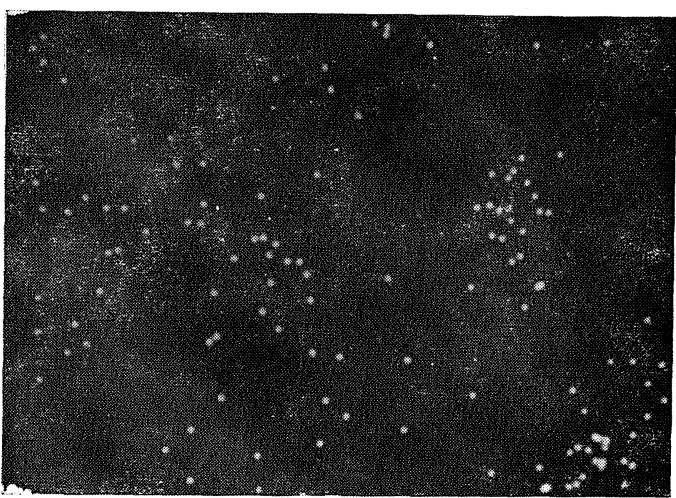

Fig. 12.-Distribución del $A l$ en la misma zona que las figuras 10,11 . Nótese la ausencia de si y Al en la zona de gran intensidad del Ca.

\section{B I B L I O G R A F I A}

(1) Le Chatelier, H.: The Constitution of Hydraulic Mortars, Mc Graw-Hill, New York (1905)

(2) Williamson, R. B.: Solidification of Portland Cement, Progress in Materials Science, Volume 15, número 3 (1972).

(3) Dron, R. Hornain, H., Petit, P.: Localisation du silicate de calcium hydraté dans les pátes de laitier granulé, C. R. Acad. Sc. Paris, tomo 280, pp. 187 (1975).

(4) KaPRANOv: El mecanismo de hidratación de los aglomerantes, Cement and Concrete Research, tomo 5, pp. $15-24$ (1975)

(5) Kittl, P. y CAstro, J.: Hidratación inicial del clínker, Materiales de Construcción, n. ${ }^{\circ}$ 158, pp. 81-85 (1975).

(6) Flint, E. P. y Wells, L. S.: J. Res. Nat. Bur. Stand, tomo 33, pp. 471 (1944). 